\title{
The Influence of Pressure on Silicon Nanowire Growth Kinetics
}

Huaizhou Zhao, Sa Zhou, Zainul Hasanali and Dunwei Wang*

Department of Chemistry, Merkert Chemistry Center, Boston College, 2609 Beacon St., Chestnut Hill, MA 02467

* All correspondence to D. Wang, email: dunwei.wang@bc.edu, Tel: 617-552-3121, Fax: $617-552-2705$ 
Figure s1. SiNWs can grow as long as $>5 \mu \mathrm{m}$ in 1 min at $500^{\circ} \mathrm{C}\left(\mathrm{P}_{\text {total }}=20\right.$ Torr $)$, suggesting no significant incubation time is involved in our experiments.

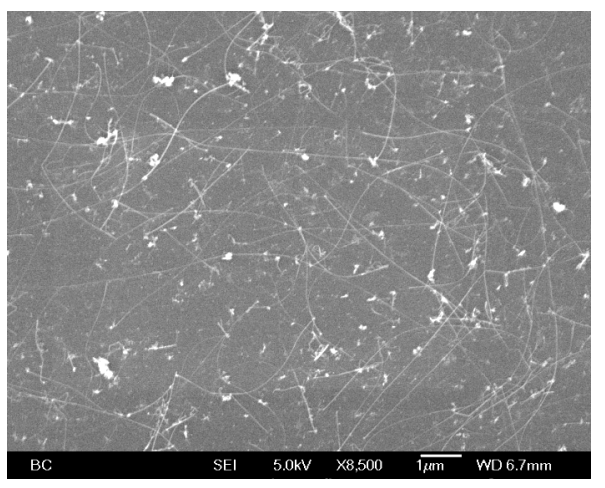


Figure s2: Growth kinetics vs. pressure under different conditions. Similar trend is followed as that for $475^{\circ} \mathrm{C}, 15 \mathrm{~nm}$ growth.

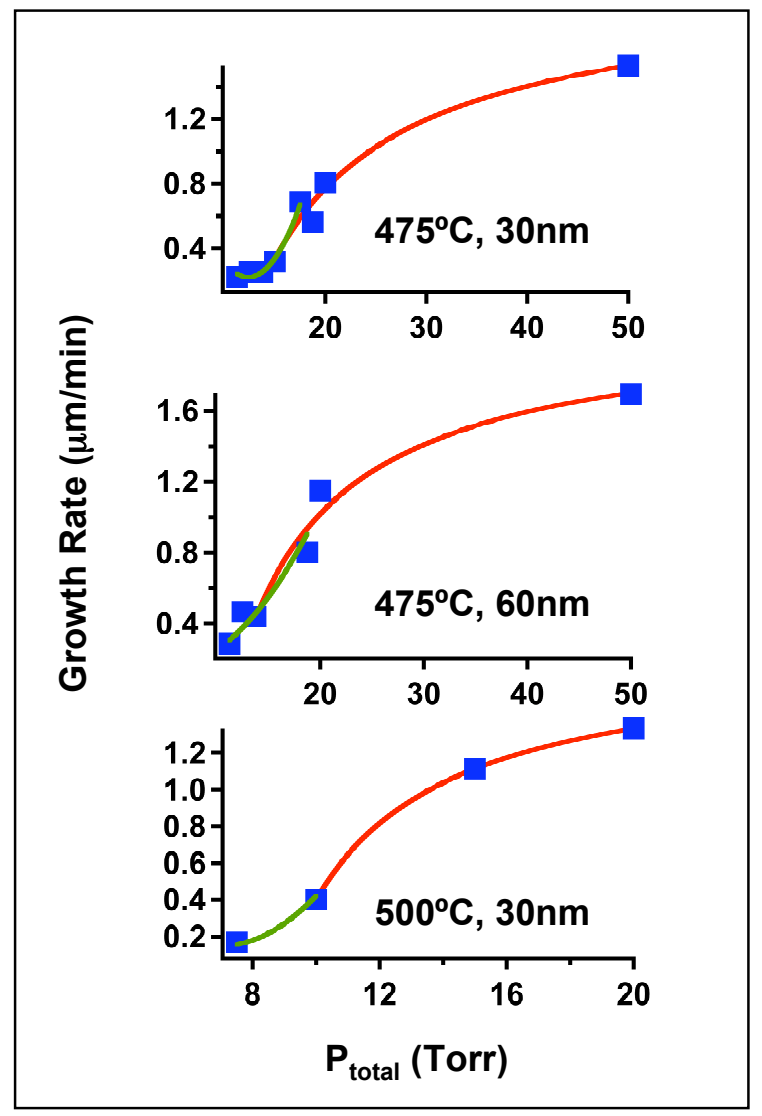


Figure s3: patterned growth, scale bar: $250 \mu \mathrm{m}$. Si only deposits where there is Au growth seed available, suggesting that spontaneous pyrolysis is negligible

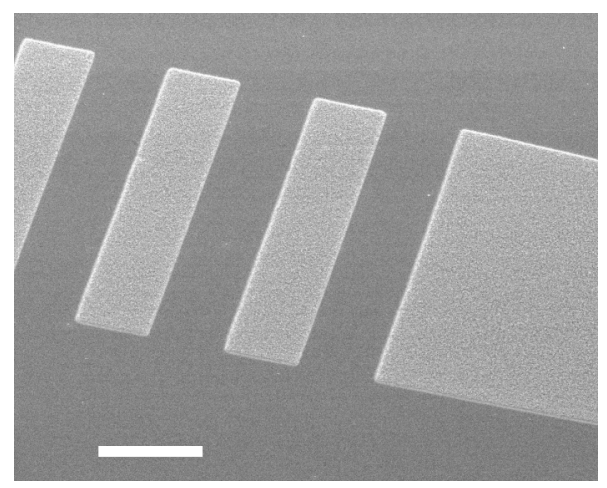


Figure s4: length measurement methodology.

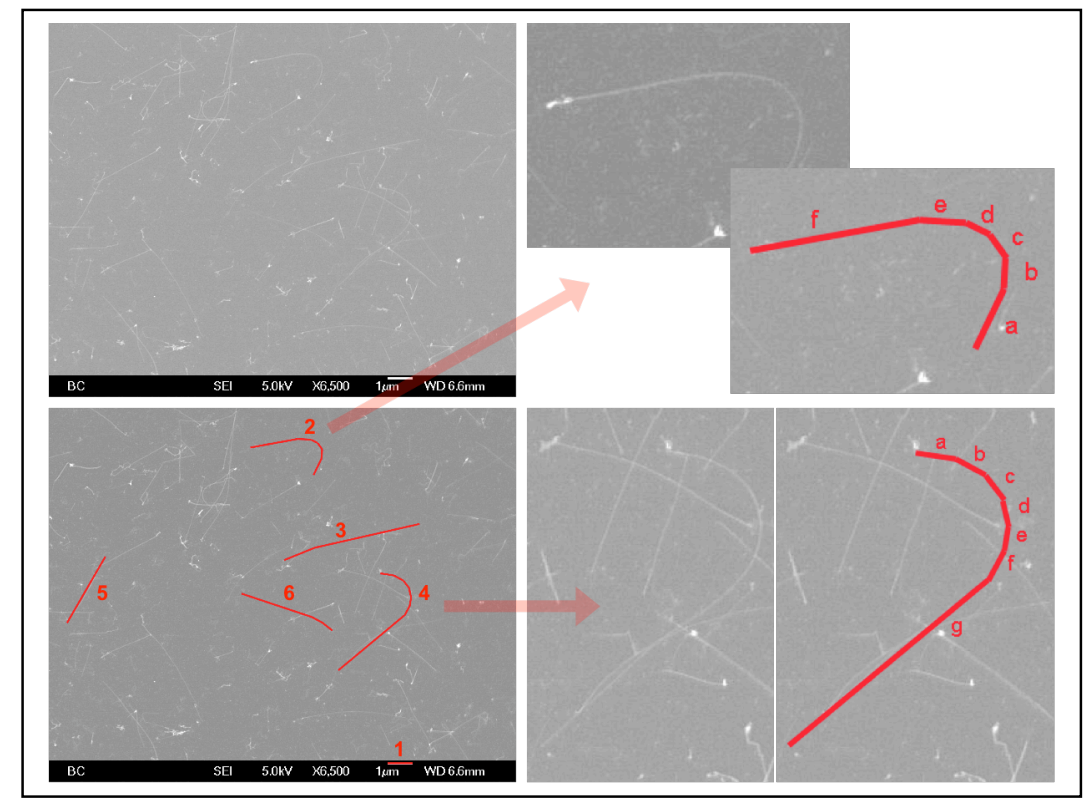

When an electron micrograph was obtained (top-left panel, a SEM picture shown in its original format), we first measured the length of the scale-bar as a means to calibrate the pixel-length correlation. Such measurements can be conveniently done using image processing softwares such as Adobe Photoshop. In this specific example, $1 \mu \mathrm{m}$ corresponds to 69 pixels in length (step 1 in the bottom-left panel picture). All nanowires with both ends visible in the view field were measured to ensure statistical accuracy. Herein we, however, only randomly chose 5 nanowires (labeled 2-6 in the bottom-left panel picture) for demonstration purposes. The length of each nanowire was then measured in the unit of pixels. From 2 to 6, the lengths are: 286, 385, 411, 210 and 271 pixels, respectively. Calibrated lengths in $\mu \mathrm{m}$ are: $4.1,5.6,6.0,3.0$ and $3.9 \mu \mathrm{m}$. The measurements are accurate for straight nanowires such as \#3, \#5 and \#6 while approximations must be taken for curved ones (\#2 and \#4). The right panel shows nanowire \#2 (top) and \#4 (bottom) in their original and approximated measurements forms. Nanowire \#2, for instance, was divided into 6 segments, labeled a-f, the length of which is: $a=51, b=24, c=22, d=20, e=35$ and $f=134$ pixels, respectively. The error introduced by the fractal measurement method is insignificant and we expect it to be much less than the statistical variations (error bar in Figure 2-4 in the main text). We have attempted to write scripts to automatically count the lengths in pixels but are unsuccessful to date. The challenge lies in the lack of a good algorithm and the relatively faint contrast of the nanowires with its surrounding background. All measurements therefore were performed manually. Three authors (H. Z., S. Z., and Z. H) conducted the length measurement independently and their results were checked by Wang for consistency. 
Figure s5: low magnification TEM pictures showing that as-grown SiNWs are uniform in diameters (i.e. without noticeable tapering).

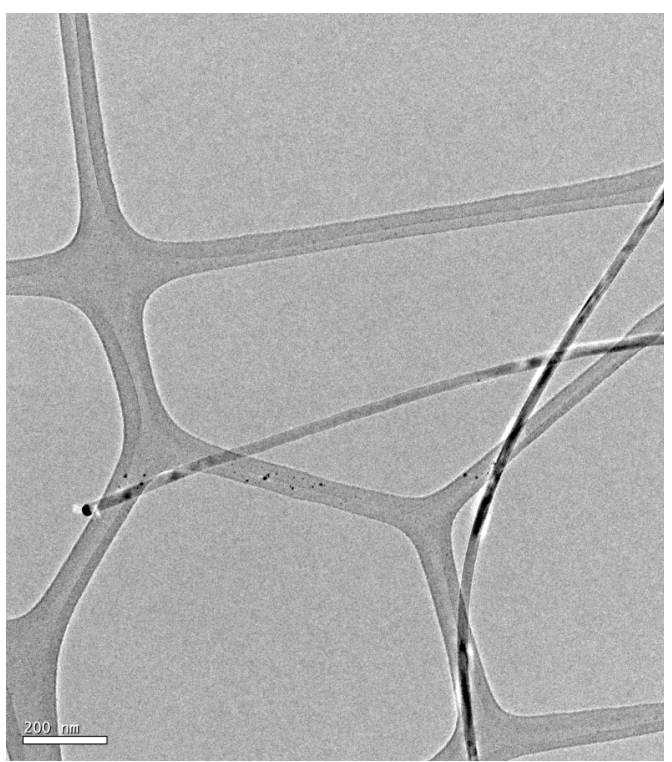

